\title{
Reduction of Fear by Intense Aerobic Exercise Approaching Physical Exhaustion
}

\author{
Newell Heywood, Susan Sabado, Bernaden De Leon \\ 2309 Langdale Avenue, Eagle Rock, California, USA \\ Email: newwick@gmail.com
}

Received May $2^{\text {nd }}, 2012$; revised June $10^{\text {th }}, 2012$; accepted July $7^{\text {th }}, 2012$

\begin{abstract}
This pilot experiment was done to test the hypothesis that physical exhaustion can cause a reduction in a person's fear level. The efficiency of intense aerobic exercise approaching physical exhaustion has been investigated as an effective part of therapy for reduction of an individual's fear reaction. The participants were 5 males and 5 females, aged 30 - 40 years old. Treatment sessions were conducted over a 1 week period where subjects first took physical check-ups to determine their physical fitness to undergo intense aerobic exercise. Subjects met in a gym within a one-week period with 3 sessions of intense aerobic exercise, with a one day rest period after each session. They took pre-test and post-tests using the Fear Questionnaire to set their current fear levels respectively. Findings showed that there is a significant change seen in the respondents in the reduction of fear through physical exhaustion by the use of intense aerobic exercises 1 .
\end{abstract}

Keywords: Endorphins; Physical Exhaustion; Strenuous Exercise; Reduction of Fear; Runner's High; Virtual Reality; Fear

\section{Introduction}

Why exhaustion? Exhaustion is a marker in the same way that street signs mark locations. A member of this research team observed that when flying terrified and facing his fear by flying anyway he was never helped the next time he flew. The next time he was still just as terrified as the time before. Then in 1984 after attending the Fear of Flying clinic at San Francisco International Airport (SFO), where they were taught to do relaxation exercises, the team member flew from SFO to Miami, Florida, doing relaxation exercises until exhaustion set in and the relaxation exercises couldn't be done any more. At that time he realized his fear had vanished. Later, on the internet he ran across an article written by some soldiers who claimed they had lost their "normal fears" after an exhausting bivouac. So that is why exhaustion became a marker. Those observations of single events, it turns out, have experimental evidence which back their validity.

Ströhie et al. (2005) demonstrated in an experiment that aerobic exercise is effective in treating panic and panic attacks. An experiment performed in 2003 demonstrated that exercise induces analgesia and sedation in the body (Sparling, Giuffrida, Piomelli, Rosskoph, \& Deitrich, 2003). Later in 2008 an experiment of "runner's high" revealed that long distance running releases endorphins into the body and a euphoric-like state happens (Boecker et al., 2008). Boecker's subjects were tested at rest and after 2 hours of endurance running $(21.5 \pm 4.7 \mathrm{~km})=$ $(13.9 \pm 2.9 \mathrm{mi})$.

So what is analgesia and sedation in the body that causes euphoria? Endorphins are natural body chemicals that occupy the same receptor sites that opium and marijuana occupy which raises the threshold of pain however there is recent evidence which suggests that the feeling of euphoria may also be caused by some other neural function. Kut et al. (2011) call the other function the "opioid-insensitive inhibitory circuit". They gave the endorphin suppressing drug, naloxone, to subjects who were feeling pain and others feeling pain whose pain thresholds were elevated by pleasure. The pain feeling group felt more pain in the absents of endorphins and the pleasure feeling group felt no pain increase in the absents of endorphins. It appears that Kut and his cohorts may have discovered a new function of physiology.

This experiment will try to explore the possible change in the fear level of the respondents by using physical exhaustion as its treatment method. Findings of this research could pave the way in furthering our understanding of the possible effects of exercise in alleviating certain mental health related problems.

\section{Method}

\section{Research Design}

This study employed a quasi-experimental approach called a "Single group pre-test post-test design" in order to assess the effectiveness of the exercise treatment with the given sample participants. This particular design is composed of a single group of participants who underwent the same evaluation measurement followed by a treatment intervention given by the researchers. After each of three experiment interventions were done, a post-test was immediately conducted to assess the effectiveness of the intervention to the participants.

\section{Participants}

This study was undertaken with 10 participants, 5 males and 5 females, ages 30 - 40 years old. Based on available data and relevant interviews, these respondents possessed significant varying degrees and diversified types of fear. Their fears include certain insects, relationship with significant people, fears 
of the unknown or future life events and many others. The participants were given honorariums to cover their expenses. The possibility that participant expectancy effects influenced reported fear reduction is a valid concern. Even though the experimental design wasn't known to the participants they should have been able to figure it out after the first experiment and that possibility cannot be guarded against in such a simple experiment.

\section{Measures}

Fear Survey Schedule П (FSS П) was developed by James H. Geer to measure the responses to commonly occurring fears. The instrument lists potential fear evoking situations and stimuli. A client rates his or her level of discomfort or distress. Each item is rated on a 7 point scale of intensity of fear. Higher scores indicate greater fear. The FSS $\Pi$ is considered a very reliable instrument with an internal consistency coefficient of .94 using Kuder Richardson formula 20.

\section{Procedures}

The participants signed informed consent forms to show their approval to join the experiment. Selection of participants was done thru purposive sampling wherein all the participants have fears as shown in the results by the Fear Survey. The ten participants took complete physical and laboratory examinations to determine their fitness to participate in the study. The tests were administered by the researchers who are practicing psychologists while the physical examination was conducted by a medical doctor.

The intense aerobics exercise was instructed by different aerobic experts in their respective fields and took place in the $35^{\text {th }}$ floor of an air-conditioned gym. The respondents underwent intense aerobic exercise sessions utilizing all equipments inside the gym up to the point of their exhaustion ( $1-3 / 2 \mathrm{hr}$ ). The 3 sessions lasted for two to three hours with an interval of one day rest period after each session. They were pre-tested before the exercise intervention was done to verify their current fear levels and were post-tested after they signaled they were exhausted, to determine the possible change of their fear level respectively, using the Fear Questionnaire.

\section{Statistical Analysis}

The Mean and Standard Deviation values were used to present the current anxiety level of the respondents as measured by the FSS $\Pi$ instrument. Also, One Way Repeated Measures ANOVA was used to test for differences in the effect of experimental interventions on the same group of subjects by examining the changes in each individual. Examining the differences between the values rather than the absolute values removes any differences due to individual responses, producing a more sensitive and more powerful test.

\section{Results}

Table 1 presents the Mean and Standard Deviation results from the initial pre-test up to the final post-test. Reflected from the findings, the initial pre-test achieved a 162.60 (34.89) mean rating which classifies the respondents as having very high levels of fears. With the application of the exercise program, results seen from post-test 1 mean of 143.30 (41.47), post-test 2
Table 1.

Mean and standard deviation findings.

\begin{tabular}{lccc}
\hline & Mean & Standard Deviation & Interpretation \\
\hline Pre-test & 162.60 & 34.89 & very high \\
Post-test 1 & 143.30 & 41.47 & high \\
Post-test 2 & 122.10 & 50.14 & average \\
Post-test 3 & 109.10 & 50.71 & average \\
\hline
\end{tabular}

mean of $122.10(50.14)$ and post-test 3 mean of 109.10 (50.71), there is a substantial decrease on the fear levels seen in the respondents $\mathrm{n}$ from High to Average.

\section{Discussion}

This experiment was meant only to be a pilot study to alert the psychological community of the reality of fearlessness produced by physical exhaustion. Its small budget dictated that only ten experimental subjects be used without a control group. It's obvious that such a small number doesn't constitute scientific proof and no valid conclusions can be drawn from it however other experiments, unknown to us at the time, demonstrate that these experimental results are correct. The other experiments demonstrate that physical exhaustion produces endorphins which cause body feelings of sedation, analgesia and euphoria which are incompatible with fear. So it turned out that not using a control group didn't hurt the results of this experiment, the results were exactly what they should have been.

Physical exhaustion, the state where a person can no longer effectively continue doing exercises, was used as the end of the intervention because other markers or procedures weren't known to us at the time. However there are other strategies that can be used. Ströhie et al. (2005) demonstrated in an experiment that aerobic exercise is effective in treating panic and panic attacks. They used aerobic exercise for 30 minutes at $70 \%$ of maximum oxygen consumption however they made no claims that endorphins were involved.

This experiment was performed in the third week of March, 2010 , in the Fitness First health center on the $35^{\text {th }}$ floor of the Wynsum building in Manila and wasn't published until 2012, however in the intervening time we discovered the experiments on runner's high which greatly strengthens these findings. Those studies have demonstrated that long distance running, which we equate to physical exhaustion, releases endorphins inducing analgesia and sedation in the body and a euphoric-like feeling happens. Thus it appears that endorphins released into the body by strenuous exercise cause a decrease in a person's fear and anxiety level which explains the success of this experiment.

It's obvious that slight or mild diseases and conditions respond best to treatment whereas severe conditions respond less favorably so. This holds true in the treatment of fear and anxiety also, which is the subject of this report. Numerous studies of cognitive behavioral therapy conclude that there is a small percentage, $10 \%$ to $15 \%$, of treated clients who get no relief from their fears at all. Thus the people who have the least fear get the best results from therapy and those who have the most fear get none at all. The other anxious people lie somewhere between those two conditions. Might it be possible that by using physical exhaustion to lessen a client's fear, before exposure therapy begins, that he may temporarily transcend out from the untreatable group into the less fearful more successfully 
treatable group?

Another possible application of exhaustion reduced fear is in the treatment of soldiers returning home with post traumatic stress disorder (PTSD). The US army is using virtual reality at Walter Reed Hospital in treating PTSD to revive soldier's repressed memories and thus make them amenable to therapy. Obviously, fear related stress so powerful that it causes repression of memories belongs in the "get no relief" (GNR) group. So when virtual reality revives their repressed memories, the soldiers are still in the GNR group. Virtual reality therapy has been reported to be $62 \%$ successful, (Reger et al., 2011). That leaves $38 \%$ unsuccessfully treated. Perhaps endorphin releasing strenuous exercise before virtual reality therapy begins will improve their success rates. How long the fearlessness state exists isn't known and needs to be determined experimentally, however endorphins in the body exist for a finite time so it would be best to do the exposure therapy immediately after exhaustion is produced.

Thus if people temporarily suspended or at least significantly reduced their fears before therapy by using endorphin releasing exercises then perhaps they would transfer out of the untreatable group and into the more successfully treatable group. An interesting variation of this procedure would be to use the elevated pain threshold activated by pleasure, in place of exhaustion, as done by Kut and his associates to see what effect that has. This experiment points to the need for more experiments to clarify the exhaustion-reduced-fear phenomena.

\section{Acknowledgements}

Susan Sabado and Bernaden De Leon designed, performed and wrote the experiment results. They are graduate psychology students at The University of Santo Tomas in Manila. They are also associated with the Philippine Mental Hospital in Manila.

Special thanks to Bella Z. Canuto who helped make this undertaking possible.

Dr. Romeo Sabado gave the participants physical exams and was present at all three sessions monitoring their health. He is the resident neurologist at the Philippine Mental Hospital.

Newell W. Heywood Jr. originated, funded, wrote the introduction and discussion, and had this report published.

\section{REFERENCES}

Boecker, H., Hendricksen, G., Koppenhoefer, M., Spilker, M. E., Spreger, T., Tolle, T. R., \& Wagner, K. J. (2008). The runner's high: Opoiddergic mechanisms in the human brain. Cerebral Cortex, 18, 2523-2531. doi:10.1093/cercor/bhn013

Kut, E., Fink, D., Folkers, G., Candia, V., von Overbeck, J., \& Pok, J. (2011). Pleasure-related analgesia activates opioid-insensitive circuits. The Journal of Neuroscience, 31, 4148-4153. doi:10.1523/JNEUROSCI.3736-10.2011

Reger, G. M., Difede, J., Gahm, G. A., Holloway, G. M., Candy, C., \& Rothbaum, B. O. (2011). Effectiveness of virtual exposure therapy for active soldiers in a military mental health clinic. Journal of Trauma Stress, 24, 93-96. doi:10.1002/jts.20574

Sparling, P. B., Giuffrida, A., Piomelli, D., Rosskoph, I., \& Deitrich, A. (2003). Exercise activates the endocannabinoid system. Neuroreport, 14, 2209-2211. doi:10.1097/00001756-200312020-00015

Ströhle, S., Feller, C., Godemann, F., Heinz, A., Onken, M., \& Dimeo, F. (2005). The acute anti-panic activity of aerobic exercise. American Journal of Psychiatry, 162, 2376-2378.

doi:10.1176/appi.ajp.162.12.2376 